\title{
Existence of Solutions for a Robin Problem Involving the $p(x)$-Laplace Operator
}

\author{
Mostafa Allaoui \\ Department of Mathematics, Faculty of sciences, Department of Mathematics, Mohamed I University, 60000 Oujda, Morocco \\ Correspondence should be addressed to Mostafa Allaoui; allaoui19@hotmail.com
}

Received 27 January 2016; Accepted 9 May 2016

Academic Editor: Patricia J. Y. Wong

Copyright (C) 2016 Mostafa Allaoui. This is an open access article distributed under the Creative Commons Attribution License, which permits unrestricted use, distribution, and reproduction in any medium, provided the original work is properly cited.

In this article we study the nonlinear Robin boundary-value problem $-\Delta_{p(x)} u=f(x, u)$ in $\Omega,|\nabla u|^{p(x)-2}(\partial u / \partial \nu)+\beta(x)|u|^{p(x)-2} u=0$ on $\partial \Omega$. Using the variational method, under appropriate assumptions on $f$, we obtain results on existence and multiplicity of solutions.

\section{Introduction}

The aim of this article is to analyze the existence of solutions of the following problem:

$$
\begin{aligned}
-\Delta_{p(x)} u & =f(x, u) \quad \text { in } \Omega, \\
|\nabla u|^{p(x)-2} \frac{\partial u}{\partial \nu}+\beta(x)|u|^{p(x)-2} u & =0 \quad \text { on } \partial \Omega,
\end{aligned}
$$

where $\Omega \subset \mathbb{R}^{N}(N \geq 2)$ is a bounded smooth domain, $\partial u / \partial v$ is the outer unit normal derivative on $\partial \Omega, p$ is a continuous function on $\bar{\Omega}$ with $p^{-}:=\inf _{x \in \bar{\Omega}} p(x)>1$, and $\beta \in L^{\infty}(\partial \Omega)$ with $\beta^{-}:=\inf _{x \in \partial \Omega} \beta(x)>0$ and $f: \Omega \times \mathbb{R} \rightarrow$ $\mathbb{R}$ is a continuous function. The main interest in studying such problems arises from the presence of the $p(x)$-Laplace operator $\operatorname{div}\left(|\nabla u|^{p(x)-2} \nabla u\right)$, which is a natural extension of the classical $p$-Laplace operator $\operatorname{div}\left(|\nabla u|^{p-2} \nabla u\right)$ obtained in the case when $p$ is a positive constant. However, such generalizations are not trivial since the $p(x)$-Laplace operator possesses a more complicated structure than $p$-Laplace operator; for example, it is inhomogeneous.

We make the following assumptions on the function $f$ :

$\left(H_{0}\right): f: \Omega \times \mathbb{R} \rightarrow \mathbb{R}$ is a continuous function and there exist two constants $C_{1}, C_{2} \geq 0$ such that

$$
|f(x, s)| \leq C_{1}+C_{2}|s|^{q(x)-1} \quad \forall(x, s) \in \Omega \times \mathbb{R},
$$

where $q(x) \in C(\Omega)$ and $1<q(x)<p^{*}(x)$ for all $x \in \Omega$.

$\left(H_{1}\right)$ : the following limit holds uniformly for a.e $x \in \Omega$ :

$$
\lim _{|s| \rightarrow \infty} \frac{f(x, s) s}{|s|^{p^{+}}}=+\infty
$$

$\left(H_{2}\right): f(x, s)=o\left(|s|^{p(x)-1}\right)$ as $s \rightarrow 0$ and uniformly for $x \in$ $\Omega$.

$\left(H_{3}\right)$ : there exist two positive constants $c_{1}$ and $c_{2}$ such that

$$
\zeta_{1}(x, s) \leq c_{1} \zeta_{1}(x, t) \leq c_{2} \zeta_{2}(x, t), \quad \forall 0 \leq s \leq t,
$$

where $\zeta_{1}(x, s)=f(x, s) s-p^{-} F(x, s)$ and $\zeta_{2}(x, s)=$ $f(x, s) s-p^{+} F(x, s)$.

By the famous Mountain Pass lemma we state the first result.

Theorem 1. Suppose that the conditions $\left(\mathrm{H}_{0}\right)-\left(\mathrm{H}_{3}\right)$ with $q^{-}>$ $p^{+}$hold. Then problem (1) has at least a nontrivial weak solution.

Assume the following hypotheses:

$\left(H_{4}\right)$ :

$$
\lim _{|s| \rightarrow 0} \frac{F(x, s)}{|s|^{p^{-}}}=\infty
$$


$\left(H_{5}\right): f(x, 0)=0$, and there exists $\delta>0$ such that $p^{-} F(x, s)-f(x, s) s>0$ for every $x \in \bar{\Omega}$ and $|s| \leq \delta$ where $F(x, s)=\int_{0}^{s} f(x, t) d t$.

$\left(H_{6}\right): f(x,-s)=-f(x, s)$, for $x \in \Omega, s \in \mathbb{R}$.

We are now in the position to state our second theorem.

Theorem 2. Suppose that $p: \bar{\Omega} \rightarrow \mathbb{R}$ is Lipschitz continuous function. Under the assumptions $\left(H_{0}\right)$ and $\left(H_{4}\right)-\left(H_{6}\right)$, problem (1) has a sequence of weak solutions $\left\{u_{n}\right\}_{n}$ such that $\left|u_{n}\right|_{L^{\infty}(\Omega)} \rightarrow$ 0 as $n \rightarrow \infty$.

For the next theorem we assume that $f$ satisfies the following conditions:

$\left(H_{7}\right): f(x, s) \geq 0$ for all $x \in \Omega$ and $s \geq 0$ with $f(x, 0) \neq 0$.

$\left(H_{8}\right): f(x, s)$ is nondecreasing with respect to $s \geq 0, \forall x \in \Omega$.

Theorem 3. Suppose that the conditions $\left(H_{0}\right),\left(H_{7}\right)$, and $\left(H_{8}\right)$ with $p \in C^{1}(\bar{\Omega})$ hold. Then problem (1) has a positive solution.

Nonlinear boundary-value problems with variable exponent have received considerable attention in recent years. This is partly due to their frequent appearance in applications such as the modeling of electrorheological fluids [1-4] and image processing [5], but these problems are very interesting from a purely mathematical point of view as well. Many results have been obtained on this kind of problems; see for example [6-13]. In [9], the authors have studied the case $f(x, u)=|u|^{p(x)-2} u$; they proved the existence of infinitely many eigenvalue sequences. Unlike the $p$-Laplacian case, for a variable exponent $p(x)$ ( $\neq$ constant), there does not exist a principal eigenvalue and the set of all eigenvalues is not closed under some assumptions. Finally, they presented some sufficient conditions that the infimum of all eigenvalues is zero and positive, respectively.

In [14], the authors obtained results on existence and multiplicity of solutions for problem (1) in the case $q^{-}>p^{+}$, under $\left(H_{0}\right)$ and the following Ambrosetti-Rabinowitz type condition:

$$
\begin{aligned}
|t| \geq M \Longrightarrow 0 \leq & \mu F(x, t) \leq f(x, t) t, \\
& \exists \mu>p^{+}, M>0 \text { such that } x \in \Omega .
\end{aligned}
$$

Here, we notice that $\left(H_{3}\right)$ is much weaker than the (AR) condition in the constant exponent case.

Very recently, the authors in [15] studied the following problem:

$$
\begin{aligned}
-\Delta_{p(x)} u & \in \lambda \partial F(x, u) \quad \text { in } \Omega, \\
|\nabla u|^{p(x)-2} \frac{\partial u}{\partial \nu}+\beta(x)|u|^{p(x)-2} u & =0 \quad \text { on } \partial \Omega,
\end{aligned}
$$

where $\lambda$ is a positive parameter, $F(x, t)$ is locally Lipschitz function in the $t$-variable integrand, and $\partial F(x, t)$ is the subdifferential with respect to the $t$-variable in the sense of Clarke. They claim that problem (6) admits at least two nontrivial solutions.
In the first result, we consider problem (1) when the nonlinear term is superlinear at infinity but does not satisfy the (AR) type condition, used in $[14,16]$, which is necessary to ensure the boundedness of the Palais-Smale (PS) type sequences of the associated functional. To overcome these difficulties, we will use the Mountain Pass Theorem [17] with Cerami condition $(C)$ which is weaker than Palais-Smale (PS) condition.

In the second result, a distinguishing feature is that we have assumed some conditions only at zero; however, there are no conditions imposed on $f$ at infinity, which is necessary in many works. Finally, in Theorem 3, applying the subsuper solution method we get a positive solution of problem (1).

This article is organized as follows. First, we will introduce some basic preliminary results and lemmas in Section 2. In Section 3, we will give the proofs of our main results.

\section{Preliminaries}

For completeness, we first recall some facts on the variable exponent spaces $L^{p(x)}(\Omega)$ and $W^{1, p(x)}(\Omega)$. For more details, see $[18,19]$. Suppose that $\Omega$ is a bounded open domain of $\mathbb{R}^{N}$ with smooth boundary $\partial \Omega$ and $p \in C_{+}(\bar{\Omega})$, where

$$
C_{+}(\bar{\Omega})=\left\{p \in C(\bar{\Omega}), \inf _{x \in \bar{\Omega}} p(x)>1\right\} .
$$

Denote by $p^{-}:=\inf _{x \in \bar{\Omega}} p(x)$ and $p^{+}:=\sup _{x \in \bar{\Omega}} p(x)$. Define the variable exponent Lebesgue space $L^{p(x)}(\Omega)$ by

$$
\begin{aligned}
& L^{p(x)}(\Omega)=\{u: \Omega \\
& \left.\quad \longrightarrow \mathbb{R} \text { is measurable and } \int_{\Omega}|u|^{p(x)} d x<+\infty\right\},
\end{aligned}
$$

with the norm

$$
|u|_{p(x)}=\inf \left\{\tau>0 ; \int_{\Omega}\left|\frac{u}{\tau}\right|^{p(x)} d x \leq 1\right\} .
$$

Define the variable exponent Sobolev space $W^{1, p(x)}(\Omega)$ by

$$
W^{1, p(x)}(\Omega)=\left\{u \in L^{p(x)}(\Omega):|\nabla u| \in L^{p(x)}(\Omega)\right\},
$$

with the norm

$$
\begin{aligned}
& \|u\|=\inf \left\{\tau>0 ; \int_{\Omega}\left(\left|\frac{\nabla u}{\tau}\right|^{p(x)}+\left|\frac{u}{\tau}\right|^{p(x)}\right) d x \leq 1\right\}, \\
& \|u\|=|\nabla u|_{p(x)}+|u|_{p(x)} .
\end{aligned}
$$

We refer the reader to $[11,18]$ for the basic properties of the variable exponent Lebesgue and Sobolev spaces.

Lemma 4 (see [19]). Both $\left(L^{p(x)}(\Omega),|\cdot|_{p(x)}\right)$ and $\left(W^{1, p(x)}(\Omega)\right.$, $\|\cdot\|)$ are separable and uniformly convex Banach spaces. 
Lemma 5 (see [19]). Hölder inequality holds, namely,

$$
\begin{aligned}
& \int_{\Omega}|u v| d x \leq 2|u|_{p(x)}|v|_{p^{\prime}(x)} \\
& \forall u \in L^{p(x)}(\Omega), v \in L^{p^{\prime}(x)}(\Omega),
\end{aligned}
$$

where $1 / p(x)+1 / p^{\prime}(x)=1$.

Lemma 6 (see [18]). Assume that the boundary of $\Omega$ possesses the cone property and $p \in C(\bar{\Omega})$ and $1 \leq q(x)<p^{*}(x)$ for $x \in$ $\bar{\Omega}$, then there is a compact embedding $W^{1, p(x)}(\Omega) \hookrightarrow L^{q(x)}(\Omega)$, where

$$
p^{*}(x)= \begin{cases}\frac{N p(x)}{N-p(x)}, & \text { if } p(x)<N ; \\ +\infty, & \text { if } p(x) \geq N .\end{cases}
$$

Now, we introduce a norm, which will be used later.

Let $\beta \in L^{\infty}(\partial \Omega)$ with $\beta^{-}:=\inf _{x \in \partial \Omega} \beta(x)>0$ and, for $u \in W^{1, p(x)}(\Omega)$, define

$$
\begin{aligned}
& \|u\|_{\beta}=\inf \{\tau \\
& \quad>0 ; \int_{\Omega}\left(\left|\frac{\nabla u}{\tau}\right|^{p(x)} d x+\int_{\partial \Omega} \beta(x)\left|\frac{u}{\tau}\right|^{p(x)}\right) d \sigma \\
& \quad \leq 1\} .
\end{aligned}
$$

Then, by Theorem 2.1 in [16], $\|\cdot\|_{\beta}$ is also a norm on $W^{1, p(x)}(\Omega)$ which is equivalent to $\|\cdot\|$.

An important role in manipulating the generalized Lebesgue-Sobolev spaces is played by the mapping defined by the following.

Lemma 7 (see [16]). Let $I(u)=\int_{\Omega}|\nabla u|^{p(x)} d x+$ $\int_{\partial \Omega} \beta(x)|u|^{p(x)} d \sigma$ with $\beta^{-}>0$. For $u \in W^{1, p(x)}(\Omega)$ one has

$$
\begin{aligned}
& \|u\|_{\beta}<1(=1,>1) \Leftrightarrow I(u)<1(=1,>1) ; \\
& \|u\|_{\beta} \leq 1 \Rightarrow\|u\|_{\beta}^{p^{+}} \leq I(u) \leq\|u\|_{\beta}^{p^{-}} ; \\
& \|u\|_{\beta} \geq 1 \Rightarrow\|u\|_{\beta}^{p^{-}} \leq I(u) \leq\|u\|_{\beta}^{p^{+}} ; \\
& \left\|u_{n}-u\right\|_{\beta} \rightarrow 0 \Leftrightarrow I\left(u_{n}-u\right) \rightarrow 0 .
\end{aligned}
$$
[20].

We recall the definition of the following condition $(C)$, see

Definition 8 (see [20]). Let $X$ be a Banach space and $J \in$ $C^{1}(X, \mathbb{R})$. Given $c \in \mathbb{R}$, one says that $J$ satisfies the Cerami $c$ condition (one denotes condition $\left(C_{c}\right)$ ) if

(i) any bounded sequence $\left\{u_{n}\right\} \subset X$ such that $J\left(u_{n}\right) \rightarrow c$ and $J^{\prime}\left(u_{n}\right) \rightarrow 0$ has a convergent subsequence;

(ii) there exist constants $\delta, R, \beta>0$ such that

$$
\left\|J^{\prime}(u)\right\|\|u\| \geq \beta
$$

$\forall u \in J^{-1}([c-\delta, c+\delta])$ with $\|u\| \geq R$.
If $J \in C^{1}(X, \mathbb{R})$ satisfies condition $\left(C_{c}\right)$ for every $c \in \mathbb{R}$, one says that $J$ satisfies condition $(C)$.

Note that condition $(C)$ is weaker than the (PS) condition. However, it was shown in [17] that from condition $(C)$ it is possible to obtain a deformation lemma, which is fundamental in order to get some min-max theorems.

Theorem 9 (see [17]). Let $X$ a Banach space, $J \in C^{1}(X, \mathbf{R})$, $e \in X$, and $r>0$, such that $\|e\|>r$ and

$$
b:=\inf _{\|u\|=r} J(u)>J(0) \geq J(e) .
$$

If J satisfies the condition $\left(C_{c}\right)$ with

$$
\begin{aligned}
c & :=\inf _{\gamma \in \Gamma t \in[0,1]} J(\gamma(t)), \\
\Gamma & :=\{\gamma \in C([0,1], X) \mid \gamma(0)=0, \gamma(1)=e\} .
\end{aligned}
$$

Then $c$ is a critical value of $J$.

Here, problem (1) is stated in the framework of the generalized Sobolev space $X:=W^{1, p(x)}(\Omega)$.

The Euler-Lagrange functional associated with (1) is defined as $J: X \rightarrow \mathbb{R}$ in

$$
\begin{aligned}
J(u)= & \int_{\Omega} \frac{1}{p(x)}|\nabla u|^{p(x)} d x+\int_{\partial \Omega} \frac{\beta(x)}{p(x)}|u|^{p(x)} d \sigma \\
& -\int_{\Omega} F(x, u) d x .
\end{aligned}
$$

One says that $u \in X$ is a weak solution of (1) if

$$
\begin{gathered}
\int_{\Omega}|\nabla u|^{p(x)-2} \nabla u \nabla v d x+\int_{\partial \Omega} \beta(x)|u|^{p(x)-2} u v d \sigma \\
-\int_{\Omega} f(x, u) v d x=0,
\end{gathered}
$$

for all $v \in X$.

Standard arguments imply that $J \in C^{1}(X, \mathbb{R})$ and

$$
\begin{aligned}
\left\langle J^{\prime}(u), v\right\rangle= & \int_{\Omega}|\nabla u|^{p(x)-2} \nabla u \nabla v d x \\
& +\int_{\partial \Omega} \beta(x)|u|^{p(x)-2} u v d \sigma \\
& -\int_{\Omega} f(x, u) v d x,
\end{aligned}
$$

for all $u, v \in X$. Thus, the weak solutions of (1) coincide with the critical points of $J$.

\section{Proof of Main Results}

For simplicity, we use $C_{i}, i=1,2, \ldots$, to denote the general positive constants whose exact values may change from line to line.

Noting that $J$ is the sum of $\left(S_{+}\right)$type map and a weaklystrongly continuous map, so $J^{\prime}$ is of $\left(S_{+}\right)$type. To see that Cerami condition $(C)$ holds, it is enough to verify that any Cerami sequence is bounded. 
Proof of Theorem 1. We check the assumption of compactness of the Mountain Pass Theorem as in the following lemma.

Lemma 10. Suppose that $\left(H_{0}\right)-\left(H_{3}\right)$ hold. If $c \in \mathbf{R}$, then any $(C)_{c}$ sequence of $J$ is bounded.

Proof. Let $\left\{u_{n}\right\}$ be a $(C)_{c}$ sequence of $J$. If $\left\{u_{n}\right\}$ is unbounded, up to a subsequence we may assume that

$$
\begin{aligned}
J\left(u_{n}\right) & \longrightarrow c, \\
\left\|u_{n}\right\|_{\beta} & \longrightarrow \infty, \\
\left\|J^{\prime}\left(u_{n}\right)\right\|\left\|u_{n}\right\|_{\beta} & \longrightarrow 0 .
\end{aligned}
$$

Let $w_{n}=\left\|u_{n}\right\|_{\beta}^{-1} u_{n}$, then $\left\{w_{n}\right\}$ is bounded in $X$; up to a subsequence we have

$$
\begin{gathered}
w_{n} \rightarrow w \quad \text { in } X, \\
w_{n} \longrightarrow w \quad \text { in } L^{q(x)}(\Omega), \\
w_{n}(x) \longrightarrow w(x) \quad \text { a.e } x \in \Omega .
\end{gathered}
$$

If $w \neq 0$, we have $J^{\prime}\left(u_{n}\right) u_{n}=0$; that is,

$$
\begin{gathered}
\int_{\Omega}\left|\nabla u_{n}\right|^{p(x)} d x+\int_{\partial \Omega} \beta(x)\left|u_{n}\right|^{p(x)} d \sigma \\
-\int_{\Omega} f\left(x, u_{n}\right) u_{n} d x=0 .
\end{gathered}
$$

Dividing (23) by $\left\|u_{n}\right\|_{\beta}^{p^{+}}$, we get

$$
\int_{\Omega} \frac{f\left(x, u_{n}\right) u_{n}}{\left\|u_{n}\right\|_{\beta}^{p^{+}}} d x<\infty
$$

On the other side, using $\left(H_{1}\right)$ and lemma of Fatou we obtain

$$
\begin{aligned}
\int_{\Omega} \frac{f\left(x, u_{n}\right) u_{n}}{\left\|u_{n}\right\|_{\beta}^{p^{+}}} d x & =\int_{\Omega} \frac{f\left(x, u_{n}\right) u_{n}\left|w_{n}\right|^{p^{+}}}{\left|u_{n}\right|^{p^{+}}} d x \\
& \longrightarrow \infty
\end{aligned}
$$

we obtain a contradiction.

If $w \equiv 0$, since $w_{n} \rightarrow 0$ in $L^{q(x)}(\Omega)$ and $|F(x, t)| \leq C(1+$ $\left.|t|^{q(x)}\right)$, by the continuity of the Nemitskii operator, we see that $F\left(\cdot, w_{n}\right) \rightarrow 0$ in $L^{1}(\Omega)$ as $n \rightarrow+\infty$; therefore,

$$
\lim _{n \rightarrow \infty} \int_{\Omega} F\left(x, w_{n}\right) d x=0 .
$$

We choose a sequence $\left\{t_{n}\right\} \subset[0,1]$ such that

$$
J\left(t_{n} u_{n}\right)=\max _{t \in[0,1]} J\left(t u_{n}\right) .
$$

Given $m>0$, since for $n$ large enough we have $\left\|u_{n}\right\|_{\beta}^{-1}\left(2 m p^{+}\right)^{1 / p^{-}} \in(0,1)$, using $(26)$ with $R=\left(2 m p^{+}\right)^{1 / p^{-}}$, we obtain

$$
\begin{aligned}
J\left(t_{n} u_{n}\right) \geq & J\left(\frac{R}{\left\|u_{n}\right\|_{\beta}} u_{n}\right)=J\left(R w_{n}\right) \\
= & \int_{\Omega} \frac{R^{p(x)}}{p(x)}\left(\left|\nabla w_{n}\right|^{p(x)}\right) d x \\
& +\int_{\partial \Omega} \frac{R^{p(x)}}{p(x)} \beta(x)\left(\left|w_{n}\right|^{p(x)}\right) d \sigma \\
& -\int_{\Omega} F\left(x, R w_{n}\right) d x \\
\geq & \frac{R^{p^{-}}}{p^{+}}-\int_{\Omega} F\left(x, R w_{n}\right) d x \geq m .
\end{aligned}
$$

That is, $J\left(t_{n} u_{n}\right) \rightarrow+\infty$, but $J(0)=0, J\left(u_{n}\right) \rightarrow c$; we see that $t_{n} \in(0,1)$ and $\left\langle J^{\prime}\left(t_{n} u_{n}\right), t_{n} u_{n}\right\rangle=\left.t_{n}(d / d t)\right|_{t=t_{n}} J\left(t u_{n}\right)=0$. It yields

$$
J\left(t_{n} u_{n}\right)-\frac{1}{p^{-}} J^{\prime}\left(t_{n} u_{n}\right) \cdot t_{n} u_{n} \longrightarrow+\infty
$$

Therefore,

$$
\begin{aligned}
& \int_{\Omega}\left(\frac{1}{p(x)}-\frac{1}{p^{-}}\right)\left|t_{n} \nabla u_{n}\right|^{p(x)} d x \\
& \quad+\int_{\partial \Omega}\left(\frac{1}{p(x)}-\frac{1}{p^{-}}\right) \beta(x)\left|t_{n} u_{n}\right|^{p(x)} d \sigma \\
& \quad+\int_{\Omega}\left(\frac{1}{p^{-}} f\left(x, t_{n} u_{n}\right)\left(t_{n} u_{n}\right)-F\left(x, t_{n} u_{n}\right)\right) d x \\
& \longrightarrow+\infty
\end{aligned}
$$

so we get

$$
\int_{\Omega}\left(\frac{1}{p^{-}} f\left(x, t_{n} u_{n}\right)\left(t_{n} u_{n}\right)-F\left(x, t_{n} u_{n}\right)\right) d x \longrightarrow+\infty .
$$

Appropriately, we have

$$
\begin{aligned}
J\left(u_{n}\right)= & J\left(u_{n}\right)-\frac{1}{p^{+}} J^{\prime}\left(u_{n}\right) \cdot u_{n} \\
= & \int_{\Omega}\left(\frac{1}{p(x)}-\frac{1}{p^{+}}\right)\left|\nabla u_{n}\right|^{p(x)} d x \\
& +\int_{\partial \Omega}\left(\frac{1}{p(x)}-\frac{1}{p^{+}}\right) \beta(x)\left|u_{n}\right|^{p(x)} d \sigma \\
& +\int_{\Omega}\left(\frac{1}{p^{+}} f\left(x, u_{n}\right) u_{n}-F\left(x, u_{n}\right)\right) d x \\
\geq & \int_{\Omega}\left(\frac{1}{p^{+}} f\left(x, u_{n}\right) u_{n}-F\left(x, u_{n}\right)\right) d x .
\end{aligned}
$$


From $\left(H_{3}\right)$, there exist two constants $c_{1}$ and $c_{2}$ such that

$$
\begin{aligned}
& J\left(u_{n}\right) \geq \int_{\Omega}\left(\frac{1}{p^{+}} f\left(x, u_{n}\right) u_{n}-F\left(x, u_{n}\right)\right) d x \\
& \geq c_{1} \int_{\Omega}\left(\frac{1}{p^{-}} f\left(x, u_{n}\right) u_{n}-F\left(x, u_{n}\right)\right) d x \\
& \quad \geq c_{1} c_{2} \int_{\Omega}\left(\frac{1}{p^{-}} f\left(x, t_{n} u_{n}\right) t_{n} u_{n}-F\left(x, t_{n} u_{n}\right)\right) d x
\end{aligned}
$$

Hence, $J\left(u_{n}\right) \rightarrow+\infty$, which is impossible and thus $\left(u_{n}\right)_{n}$ is bounded in $X$. try.

We will show that $J$ possesses the Mountain Pass geome-

Lemma 11. Under the conditions $\left(H_{0}\right)-\left(H_{2}\right)$, there exist $r>0$ and $\tau$ such that $J(u)>\tau$ when $\|u\|_{\beta}=r$.

Proof. In view of $\left(H_{0}\right)$ and $\left(H_{2}\right)$, there exists $C_{1}>0$ such that

$$
|F(x, t)| \leq \frac{1}{2 p^{+}}|t|^{p(x)}+C_{1}|t|^{q(x)},
$$

$$
\text { for }(x, t) \in \Omega \times \mathbf{R} \text {. }
$$

Therefore, for $\|u\|_{\beta} \leq 1$ we have

$$
\begin{aligned}
J(u) \geq & \frac{1}{p^{+}} \int_{\Omega}|\nabla u|^{p(x)} d x+\frac{1}{p^{+}} \int_{\partial \Omega} \beta(x)|u|^{p(x)} d \sigma \\
& -\frac{1}{2 p^{+}} \int_{\Omega}|u|^{p(x)} d x-C_{1} \int_{\Omega}|u|^{q(x)} d x \\
\geq & \frac{C_{2}}{2 p^{+}} \int_{\Omega}|\nabla u|^{p(x)} d x+\frac{C_{2}}{2 p^{+}} \int_{\partial \Omega} \beta(x)|u|^{p(x)} d \sigma \\
& -C_{1} \int_{\Omega}|u|^{q(x)} d x \geq \frac{C_{2}}{2 p^{+}}\|u\|_{\beta}^{p^{+}}-C_{3}\|u\|_{\beta}^{q^{-}} \\
\geq & \|u\|_{\beta}^{p^{+}}\left(\frac{C_{2}}{2 p^{+}}-C_{3}\|u\|_{\beta}^{q^{-}-p^{+}}\right) .
\end{aligned}
$$

Since $p^{+}<q^{-}$, the function $t \mapsto\left(C_{2} / 2 p^{+}-C_{3} t^{q^{-}-p^{+}}\right)$is strictly positive in a neighborhood of zero. It follows that there exist $r>0$ and $\tau>0$ such that

$$
J(u) \geq \tau \quad \forall u \in X:\|u\|=r
$$

To apply the Mountain Pass Theorem, it suffices to show that

$$
J(t u) \longrightarrow-\infty \quad \text { as } t \longrightarrow+\infty
$$

for a certain $u \in X$.

Let $u \in X \backslash\{0\}$; by $\left(H_{1}\right)$, we can choose a constant $A>\left(\int_{\Omega}(1 / p(x))|\nabla u|^{p(x)} d x+\int_{\partial \Omega}(\beta(x) / p(x))|u|^{p(x)} d \sigma\right) /$ $\int_{\Omega}|u|^{p^{+}} d x$, such that

$$
F(x, t) \geq A|t|^{p^{+}} \quad \text { uniformly in } x \in \Omega .
$$

Let $t>1$ be large enough; we have

$$
\begin{aligned}
J(t u) & \int_{\Omega} \frac{t^{p(x)}}{p(x)}|\nabla u|^{p(x)} d x+\int_{\partial \Omega} \frac{t^{p(x)} \beta(x)}{p(x)}|u|^{p(x)} d \sigma \\
& -\int_{\Omega} F(x, t u) d x \\
\leq & t^{p^{+}}\left(\int_{\Omega} \frac{1}{p(x)}|\nabla u|^{p(x)} d x+\int_{\partial \Omega} \frac{\beta(x)}{p(x)}|u|^{p(x)} d \sigma\right) \\
& -\int_{|t u|>C_{A}} F(x, t u) d x-\int_{|t u| \leq C_{A}} F(x, t u) d x \\
\leq & t^{p^{+}}\left(\int_{\Omega} \frac{1}{p(x)}|\nabla u|^{p(x)} d x+\int_{\partial \Omega} \frac{\beta(x)}{p(x)}|u|^{p(x)} d \sigma\right) \\
& -A t^{p^{+}} \int_{\Omega}^{|u|^{p^{+}}} d x-\int_{|t u| \leq C_{A}} F(x, t u) d x \\
& +A t^{p^{+}} \int_{|t u| \leq C_{A}}|u|^{p^{+}} d x \\
& -A t^{p^{+}} \int_{\Omega}|u|^{p^{+}} d x+C_{1}, \\
\leq & t^{p^{+}}\left(\int_{\Omega} \frac{1}{p(x)}|\nabla u|^{p(x)} d x+\int_{\partial \Omega} \frac{\beta(x)}{p(x)}|u|^{p(x)} d \sigma\right)
\end{aligned}
$$

where $C_{1}>0$ is a constant, which implies that

$$
J(t u) \longrightarrow-\infty \quad \text { as } t \longrightarrow+\infty \text {. }
$$

It follows that there exists $e \in X$ such that $\|e\|_{\beta}>r$ and $J(e)<0$. According to the Mountain Pass Theorem, $J$ admits a critical value $c$ which is characterized by

$$
c=\inf _{\gamma \in \Gamma} \sup _{t \in[0,1]} J(\gamma(t))
$$

where

$$
\Gamma=\{\gamma \in C([0,1], X): \gamma(0)=0, \gamma(1)=e\} .
$$

This completes the proof.

Proof of Theorem 2. The main idea (developed by Wang [21]) is to extend $f \in C(\Omega \times(-\epsilon, \epsilon), \mathbb{R})$ to an appropriate function $\tilde{f} \in C(\Omega \times \mathbb{R}, \mathbb{R})$ in order to prove for the associated modified functional $\bar{J}$ the existence of a sequence of weak solutions tending to zero in $L^{\infty}$ norm. Therefore, it is worth recalling the following proposition.

Proposition 12 (see [22]). Let $J \in C^{1}(X, \mathbb{R})$, where $X$ is a Banach space. Assume that $J$ satisfies the (PS) condition and is even and bounded from below, and $J(0)=0$. If for any $n \in \mathbb{N}$, there exists a $k$-dimensional subspace $X_{n}$ and $\rho_{n}>0$ such that

$$
\sup _{X_{n} \cap S_{\rho_{k}}} J<0 \text {, }
$$


where $S_{\rho}:=\{u \in X:\|u\|=\rho\}$, then $J$ has a sequence of critical values $c_{n}<0$ satisfying $c_{n} \rightarrow 0$ as $n \rightarrow \infty$.

We need to state the following results.

Claim 1. When $J(u)=J^{\prime}(u) \cdot u=0$, then $u=0$.

Indeed, suppose that $u \neq 0$. Thus

$$
\begin{aligned}
& \int_{\Omega} F(x, u) d x \\
& \quad=\int_{\Omega} \frac{1}{p(x)}|\nabla u|^{p(x)} d x+\int_{\partial \Omega} \frac{\beta(x)}{p(x)}|u|^{p(x)} d \sigma, \\
& \int_{\Omega}|\nabla u|^{p(x)} d x+\int_{\partial \Omega} \beta(x)|u|^{p(x)} d \sigma \\
& \quad=\int_{\Omega} f(x, u) u d x .
\end{aligned}
$$

Then we obtain

$$
\begin{aligned}
& p^{-} \int_{\Omega} F(x, u) d x \\
& \quad=p^{-}\left(\int_{\Omega} \frac{1}{p(x)}|\nabla u|^{p(x)} d x+\int_{\partial \Omega} \frac{\beta(x)}{p(x)}|u|^{p(x)} d \sigma\right) \\
& \quad \leq \int_{\Omega} f(x, u) u d x
\end{aligned}
$$

which contradicts the assumption $\left(H_{5}\right)$.

Claim 2. There exist $\delta>0$ and $\tilde{f} \in C(\Omega \times \mathbb{R})$ such that $\tilde{f}$ is odd and

$$
\begin{aligned}
& f(x, t)=\tilde{f}(x, t) \quad \text { for }|t|<\delta, \\
& p^{-} \widetilde{F}(x, t)-\tilde{f}(x, t) t \geq 0, \quad \forall(x, t) \in \Omega \times \mathbb{R}, \\
& p^{-\widetilde{F}}(x, t)-\widetilde{f}(x, t) t=0, \quad \text { for }|t|>\delta \quad(\text { or } t=0),
\end{aligned}
$$

where $\widetilde{F}(x, t)=\int_{0}^{t} \tilde{f}(x, s) d s$.

In fact, let us define $\widetilde{F}(x, t)=h(t) F(x, t)+C_{1}(1-h(t))|t|^{p^{-}}$ where $C_{1}$ is a positive constant and $h$ is a cut-off function presented as follows:

$$
\begin{aligned}
& h(t)= \begin{cases}1, & \text { if }|t| \leq \frac{\delta}{2} \\
0, & \text { if }|t| \geq \delta\end{cases} \\
& h^{\prime}(t) t \leq 0 \\
&\left|h^{\prime}(t)\right| \leq \frac{4}{\delta} .
\end{aligned}
$$

For $|t| \leq \delta / 2$, (46) easily holds.

On the other hand, we have

$$
\begin{aligned}
\widetilde{f}(x, t)= & \frac{\partial}{\partial t} \widetilde{F}(x, t) \\
= & h^{\prime}(t) F(x, t)+h(t) f(x, t) \\
& +C_{1}(1-h(t))\left(|t|^{p^{-}}\right)^{\prime}-C_{1} h^{\prime}(t)|t|^{p^{-}} .
\end{aligned}
$$

It is easy to check that for $|t| \geq \delta$ we have

$$
p^{-} \widetilde{F}(x, t)=C_{1} p^{-}|t|^{p^{-}}
$$

Hence, (48) is satisfied. In the rest, from $\left(H_{4}\right)$, we can choose $\delta>0$ small enough to get $F(x, t) \geq C_{1}|t|^{p^{-}}$when $t \in[\delta / 2, \delta]$ and the formula (47) holds since $h^{\prime}(t) t \leq 0$.

Claim 3. The associated modified functional $\bar{J}$ satisfies the Palais-Smale condition.

In fact, by Claim 2 , it is easy to see that $\bar{J}$ is even and $\bar{J} \epsilon$ $C^{1}(X, \mathbb{R})$. For $\|u\|_{\beta}>1$, we have

$$
\begin{aligned}
\bar{J}= & \int_{\Omega} \frac{1}{p(x)}|\nabla u|^{p(x)} d x+\int_{\partial \Omega} \frac{\beta(x)}{p(x)}|u|^{p(x)} d \sigma \\
& -\int_{\Omega} \widetilde{F}(x, u) d x \\
\geq & \frac{1}{p^{+}}\left(\int_{\Omega}|\nabla u|^{p(x)} d x+\int_{\partial \Omega} \beta(x)|u|^{p(x)} d \sigma\right) \\
& -A \int_{\Omega}|u|^{p^{-}} d x .
\end{aligned}
$$

Because $X \hookrightarrow L^{p^{-}}(\Omega)$ with $A$ is a positive constant, $\bar{J}$ is coercive, that is, $\bar{J} \rightarrow+\infty$ as $\|u\|_{\beta} \rightarrow+\infty$. Hence, to verify that $\bar{J}$ satisfies (PS) condition on $X$, it is enough to verify that any (PS) sequence is bounded. Hence, by the coercivity of $\bar{J}$, any (PS) sequence is bounded in $X$.

Next, we modify and extend $f(x, u)$ to get $\tilde{f}(x, u) \in$ $C(\bar{\Omega} \times \mathbb{R})$ satisfying the assertions of Proposition 12 .

For any $k \in \mathbb{N}$ we have $k$ independent smooth functions $e_{i}$ for $i=1,2, \ldots, k$, and define the subspace $X_{k}:=$ $\operatorname{span}\left\{e_{1}, \ldots, e_{k}\right\}$.

From Claim 2, for $\|u\|_{\beta}<1$ we can obtain

$$
\begin{aligned}
\bar{J} \leq & \frac{1}{p^{-}}\left(\int_{\Omega}|\nabla u|^{p(x)} d x+\int_{\partial \Omega} \beta(x)|u|^{p(x)} d \sigma\right) \\
& -C \int_{\Omega}|u|^{p^{-}} d x \leq \frac{1}{p^{-}}\|u\|_{\beta}^{p^{-}}-C \int_{\Omega}|u|^{p^{-}} d x .
\end{aligned}
$$

By (53) and as it is well known that all norms in $X_{k}$ are equivalent, for sufficiently small $\rho_{k}$ and suitable positive constant $C$ we obtain

$$
\sup _{X_{k} \cap S_{\rho_{n}}} \bar{J}<0 .
$$

As a consequence of this fact, we observe that the conditions of Proposition 12 hold and thus there exists a sequence of negative critical values $c_{k}$ for the functional $\bar{J}$ such that $c_{k} \rightarrow 0$ as $k \rightarrow \infty$.

Afterwards, for any $u_{k} \in X$ satisfying $\bar{J}\left(u_{k}\right)=c_{k}$ and $\bar{J}^{\prime}\left(u_{k}\right)=0,\left\{u_{k}\right\}_{k}$ is (PS) $)_{0}$ sequence of $\bar{J}$. Passing, if necessary, to a subsequence still denoted by $\left\{u_{k}\right\}_{k}$, we may suppose that $\left\{u_{k}\right\}_{k}$ has a limit. 
From Claims 1 and 2 it is clear that 0 is the only critical point when the energy is zero and thus $\left\{u_{k}\right\}_{k}$ converges to 0 . It follows from $[23,24]$ that

$$
\begin{aligned}
\left\{u_{k}\right\}_{k} & \in C(\bar{\Omega}), \\
\left|u_{k}\right|_{L^{\infty}(\Omega)} & \longrightarrow 0
\end{aligned}
$$

as $k \longrightarrow \infty$.

So in view of Claim 2, we have $\left|u_{k}\right|_{C(\Omega)} \leq \delta / 2$. Thereby, the sequences $\left\{u_{k}\right\}_{k}$ are solutions of problem (1).

Proof of Theorem 3. Firstly, we recall the definition of subsupersolution of problem (1) as follows. We call $u \in X$ a subsolution (resp. supersolution) of (1) if, for every $v \in X$ with $v \geq 0$,

$$
\begin{aligned}
& \int_{\Omega}|\nabla u|^{p(x)-2} \nabla u \nabla v d x+\int_{\partial \Omega} \beta(x)|u|^{p(x)-2} u v d \sigma \\
& \quad \leq(\text { resp. } \geq) \int_{\Omega} f(x, u) v d x .
\end{aligned}
$$

Lemma 13. Let $p \in C^{1}(\bar{\Omega})$. Suppose that $f$ satisfies the subcritical growth condition $\left(H_{0}\right)$ and the function $f(x, t)$ is nondecreasing in $t \in \mathbb{R}$. If there exist a subsolution $u_{0} \in$ $W^{1, p(x)}(\Omega) \cap L^{\infty}(\Omega)$ and a supersolution $v^{0} \in W^{1, p(x)}(\Omega) \cap$ $L^{\infty}(\Omega)$ of (1) such that $u_{0} \leq v^{0}$, then (1) has a minimal solution $\underline{u}$ and a maximal solution $\bar{v}$ in the order interval $\left[u_{0}, v^{0}\right]$ (i.e., $\left.u_{0} \leq \underline{u} \leq \bar{v} \leq v^{0}\right)$.

The proof of Lemma 13 is built on the fixed point theory for the increasing operator on the order interval (see e.g., [25]) and is similar to that given in [26] for the $p(x)$-Laplacian case.

According to Proposition 2.2 in [15], the mapping $I^{\prime}$ : $X \rightarrow X^{*}$ such that for all $u, v$ in $X ;\left\langle I^{\prime}(u), v\right\rangle=$ $\int_{\Omega}|\nabla u|^{p(x)-2} \nabla u \nabla v d x+\int_{\partial \Omega} \beta(x)|u|^{p(x)-2} u v d \sigma$ is a strictly monotone, bounded homeomorphism, and consequently we have the following.

Proposition 14. Let $q \in C(\bar{\Omega})$ with $1<q(x)<p^{*}(x)$ for $x \in \bar{\Omega}$, then for $g \in L^{(q(x)) /(q(x)-1)}(\Omega)\left(\right.$ or $\left.g \in C^{0, \alpha}(\Omega)\right)$, the problem

$$
\begin{aligned}
-\Delta_{p(x)} u & =g(x), \quad \text { in } \Omega ; \\
|\nabla u|^{p(x)-2} \frac{\partial u}{\partial \nu}+\beta(x)|u|^{p(x)-2} u & =0, \quad \text { on } \partial \Omega,
\end{aligned}
$$

has a unique solution $u$ in $X$.

Let us consider the following problem:

$$
\begin{aligned}
-\Delta_{p(x)} u & =M, \quad \text { in } \Omega ; \\
|\nabla u|^{p(x)-2} \frac{\partial u}{\partial \nu}+\beta(x)|u|^{p(x)-2} u & =0, \quad \text { on } \partial \Omega,
\end{aligned}
$$

with $M>0$. By Proposition 14, the strong maximum principle [27] and the result of regularity in [28], problem $\left(\mathscr{P}_{2}\right)$ has a unique positive solution $u_{1}$ such that $u_{1}(x)>0$ for each $x \in \bar{\Omega}$. have

Taking $M=\sup _{x \in \bar{\Omega}} f(x, t)$, for any $v \in X$ with $v \geq 0$ we

$$
\begin{aligned}
& \int_{\Omega}\left|\nabla u_{1}\right|^{p(x)-2} \nabla u_{1} \nabla v d x+\int_{\partial \Omega} \beta(x)\left|u_{1}\right|^{p(x)-2} u_{1} v d \sigma \\
& =\int_{\Omega} M v d x \geq \int_{\Omega} f\left(x, u_{1}\right) v d x .
\end{aligned}
$$

Hence, $u_{1}$ is a positive supersolution of problem (1).

Obviously 0 is a subsolution of (1). By Lemma 13, (1) has a solution $u \in\left[0, u_{1}\right]$.

\section{Competing Interests}

The author declares that he has no competing interests.

\section{References}

[1] T. G. Myers, "Thin films with high surface tension," SIAM Review, vol. 40, no. 3, pp. 441-462, 1998.

[2] M. Rǔžicka, Electrorheological Fluids: Modeling and Mathematical Theory, Springer, Berlin, Germany, 2000.

[3] V. V. Zhikov, "Averaging of functionals of the calculus of variations and elasticity theory," Mathematics of the USSRIzvestiya, vol. 29, no. 1, pp. 33-66, 1987.

[4] V. V. Zhikov, S. M. Kozlov, and O. A. Oleinik, Homogenization of Differential Operators and Integral Functionals, Springer, Berlin, Germany, Translated from Russian by G. A. Yosifian, 1994.

[5] Y. M. Chen, S. Levine, and M. Rao, "Variable exponent, linear growth functionals in image restoration," SIAM Journal on Applied Mathematics, vol. 66, no. 4, pp. 1383-1406, 2006.

[6] M. Allaoui, "Existence of solutions for a Robin problem involving the $p(x)$-Laplacian," Applied Mathematics E-Notes, vol. 14, pp. 107-115, 2014.

[7] M. Allaoui, "Continuous spectrum of steklov nonhomogeneous elliptic problem," Opuscula Mathematica, vol. 35, no. 6, pp. 853866, 2015.

[8] J. Chabrowski and Y. Fu, "Existence of solutions for $p(x)$ Laplacian problems on a bounded domain," Journal of Mathematical Analysis and Applications, vol. 306, no. 2, pp. 604-618, 2005.

[9] S.-G. Deng, Q. Wang, and S. Cheng, "On the $p(x)$-Laplacian Robin eigenvalue problem," Applied Mathematics and Computation, vol. 217, no. 12, pp. 5643-5649, 2011.

[10] S.-G. Deng, "A local mountain pass theorem and applications to a double perturbed $p(x)$-Laplacian equations," Applied Mathematics and Computation, vol. 211, no. 1, pp. 234-241, 2009.

[11] X. Shi and X. Ding, "Existence and multiplicity of solutions for a general $p(x)$-Laplacian Neumann problem," Nonlinear Analysis: Theory, Methods \& Applications, vol. 70, no. 10, pp. 3715-3720, 2009.

[12] A. Ourraoui, "Multiplicity results for Steklov problem with variable exponent," Applied Mathematics and Computation, vol. 277, pp. 34-43, 2016.

[13] L.-L. Wang, Y.-H. Fan, and W.-G. Ge, "Existence and multiplicity of solutions for a Neumann problem involving the $p(x)$-Laplace operator," Nonlinear Analysis: Theory, Methods and Applications, vol. 71, no. 9, pp. 4259-4270, 2009.

[14] N. Tsouli and O. Darhouche, "Existence and multiplicity results for nonlinear problems involving the $p(x)$-Laplace operator," Opuscula Mathematica, vol. 34, no. 3, pp. 621-638, 2014. 
[15] B. Ge and Q.-M. Zhou, "Multiple solutions for a Robin-type differential inclusion problem involving the $p(x)$-Laplacian," Mathematical Methods in the Applied Sciences, 2013.

[16] S.-G. Deng, "Positive solutions for Robin problem involving the $p(x)$-Laplacian," Journal of Mathematical Analysis and Applications, vol. 360, no. 2, pp. 548-560, 2009.

[17] P. Bartolo, V. Benci, and D. Fortunato, "Abstract critical point theorems and applications to some nonlinear problems with 'strong' resonance at infinity,' Nonlinear Analysis, vol. 7, no. 9, pp. 981-1012, 1983.

[18] X. L. Fan, J. S. Shen, and D. Zhao, "Sobolev embedding theorems for spaces $W^{k, p(x)}(\Omega)$," Journal of Mathematical Analysis and Applications, vol. 262, no. 2, pp. 749-760, 2001.

[19] X. L. Fan and D. Zhao, "On the spaces $L^{p(x)}(\Omega)$ and $W^{m, p(x)}$ $(\Omega)$," Journal of Mathematical Analysis and Applications, vol. 263, no. 2, pp. 424-446, 2001.

[20] G. Cerami, "An existence criterion for the critical points on unbounded manifolds," Istituto Lombardo Accademia di Scienze e Lettere Rendiconti A, vol. 112, no. 2, pp. 332-336, 1978.

[21] Z.-Q. Wang, "Nonlinear boundary value problems with concave nonlinearities near the origin," Nonlinear Differential Equations and Applications, vol. 8, no. 1, pp. 15-33, 2001.

[22] H.-P. Heinz, "Free Ljusternik-Schnirelman theory and the bifurcation diagrams of certain singular nonlinear problems," Journal of Differential Equations, vol. 66, no. 2, pp. 263-300, 1987.

[23] X. L. Fan and D. Zhao, "A class of De Giorgi type and Hölder continuity," Nonlinear Analysis: Theory, Methods \& Applications, vol. 36, no. 3, pp. 295-318, 1999.

[24] X. Fan, "Global C1, $\alpha$ regularity for variable exponent elliptic equations in divergence form," Journal of Differential Equations, vol. 235, no. 2, pp. 397-417, 2007.

[25] H. Amann, "Fixed point equations and nonlinear eigenvalue problems in ordered banach spaces," SIAM Review, vol. 18, no. 4, pp. 620-709, 1976.

[26] X. L. Fan, "On the sub-supersolution method for $p(x)$ Laplacian equations," Journal of Mathematical Analysis and Applications, vol. 330, no. 1, pp. 665-682, 2007.

[27] X. L. Fan, Y. Z. Zhao, and Q. H. Zhang, "A strong maximum principle for $p(x)$-Laplace equations," Chinese Journal of Contemporary Mathematics, vol. 24, pp. 277-282, 2003.

[28] X. Fan, "Global $C^{1, \alpha}$ regularity for variable exponent elliptic equations in divergence form," Journal of Differential Equations, vol. 235, no. 2, pp. 397-417, 2007. 


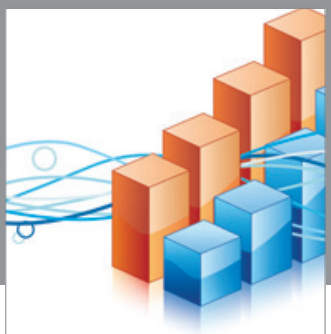

Advances in

Operations Research

vatem alat4

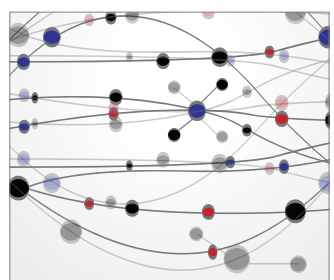

\section{The Scientific} World Journal
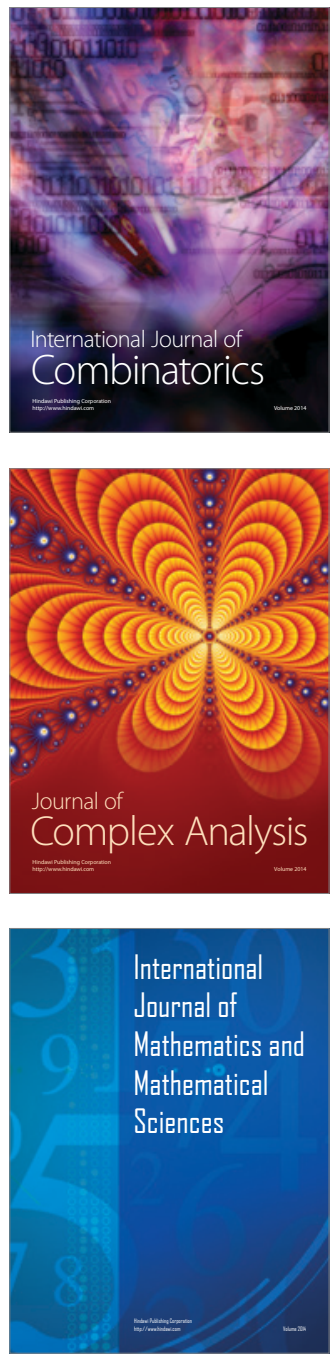
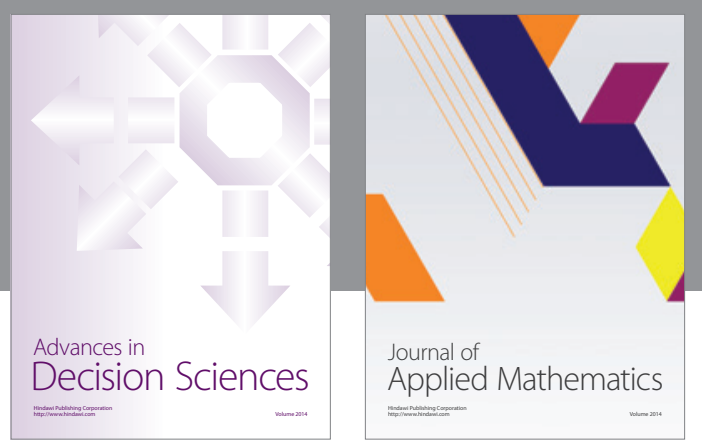

Algebra

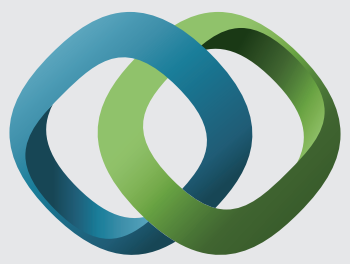

\section{Hindawi}

Submit your manuscripts at

http://www.hindawi.com
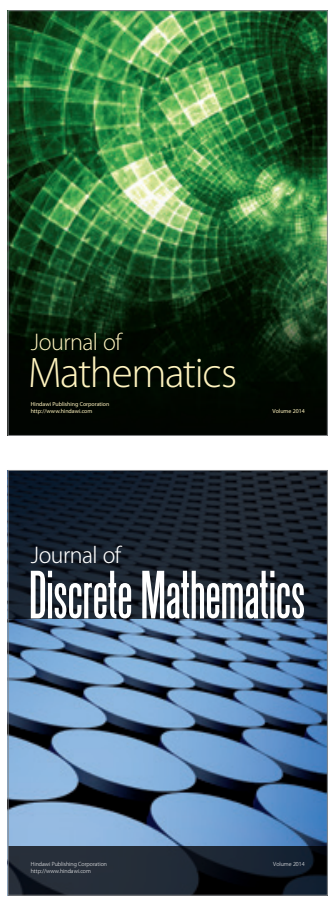

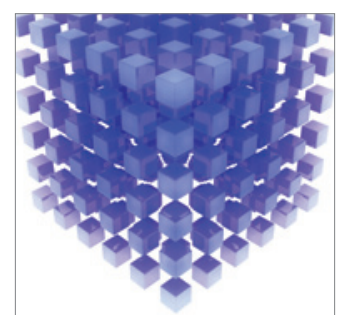

Mathematical Problems in Engineering
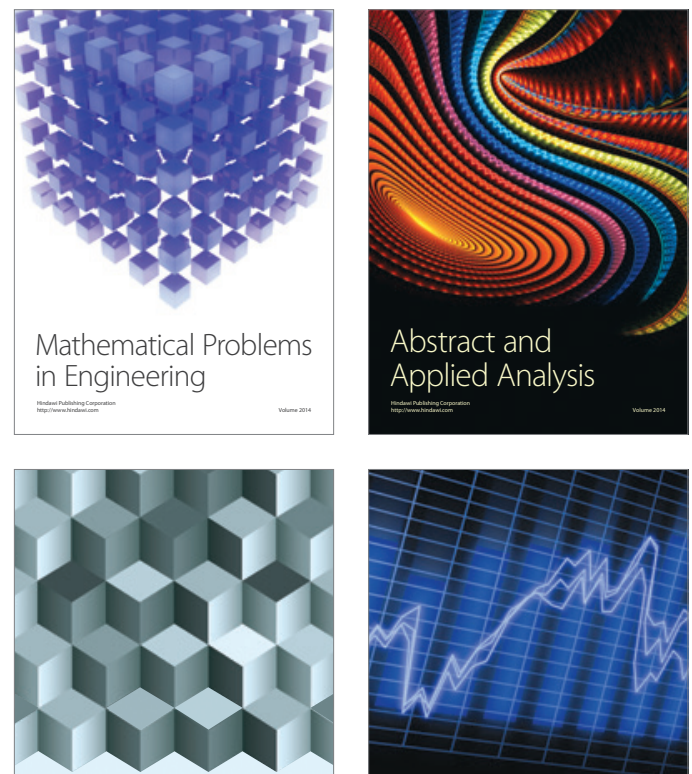

Journal of

Function Spaces

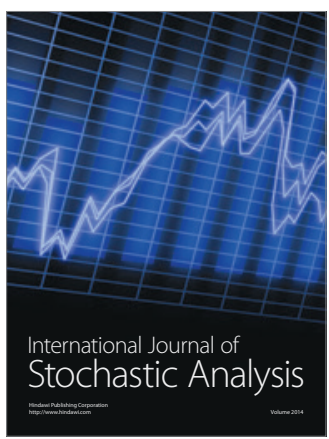

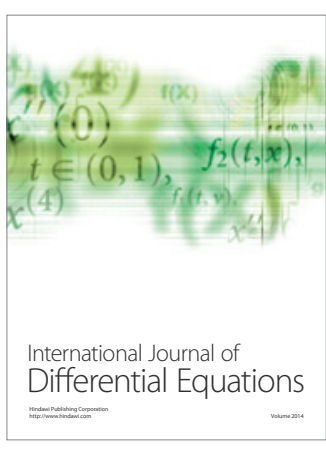
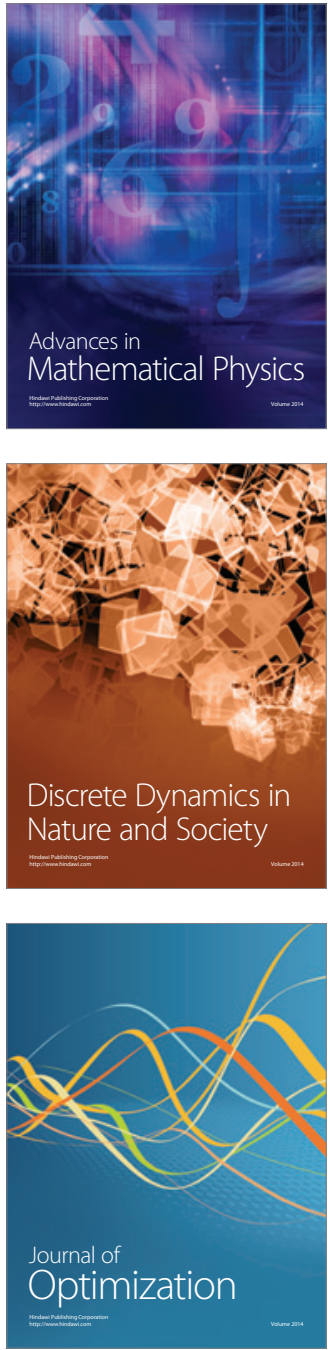